\title{
PREDICTIVE CONTROL SYSTEMS IN CONSTRUCTION EQUIPMENT
}

\author{
Mirosław Skibniewski ${ }^{a}$, Stanisław Michałowski ${ }^{\mathrm{b}}$, Andrzej Sobczyk ${ }^{\mathrm{c}}$ \\ ${ }^{a}$ Purdue University, West Lafayette, Indiana, USA; \\ IMBiGS - Institute for Mechanized Construction and Rock Mining, Warsaw, Poland \\ ${ }^{\mathrm{b}}$ Cracow University of Technology, Kraków, Poland; e-mail: pmmichal@cyf-kr.edu.pl \\ ${ }^{\mathrm{c}}$ Cracow University of Technology, Kraków, Poland
}

\begin{abstract}
In surface grading operations the final surface profile depends on the series of the preceding tasks. For example, when the road surface is levelled with the use of a numerically controlled grader, it is necessary to consider subsequent compacting tasks as well, as the final result should include a uniform compaction of the soil. Similarly, in the case of laser-control asphalt pavers or pavers with flexible connectors, with a thicker asphalt layer being applied one ought to consider subsequent compaction of the asphalt. The construction process control in these types of cases should take into account the subsequent tasks leading to the desired surface finishing effect. It is also necessary to determine the effects of each task, based on the initial physical characteristics of the material such as soil or asphalt, with respect to the specified targeted parameters of the material. In case of the compaction of soil or asphalt, there is sufficient experiential knowledge of the resulting changes of the physical parameters of the compacted medium due to the passage of static or vibration rollers with specified parameters. This assumption makes it possible to control the tasks of the control regulators with the use of specialized programs installed in on-board computers in graders or pavers. The use of such programs will allow for efficient management of relevant operational parameters related to surface finish quality. A general concept of such control system is presented herein.
\end{abstract}

Keywords: road construction, earthworks, asphalt applications, compaction vibrations, surface levelling, automatic controls

\section{BACKGROUND AND INTRODUCTION}

Defects in asphalt surface quality are very often related to inadequate compaction [1]. Modern asphalt pavers are equipped with self-leveling floating screeds. Screeds employ different design solutions to pre-compact the material. The choice between a tamping or a vibrating screed and a combination of the two is often related to the required final density factor. All asphalt compaction systems are available in either fixed or telescoping versions.

Tamping screeds use a tamping mechanism that operates with a vertical or inclined high-amplitude oscillating movement at comparatively low frequencies. Only a thin strip of the tamping unit is in contact with the asphalt mixes that results in high impact pressure on the material being laid. A static plate follows the tamping unit.

Vibrating screeds work with higher frequencies and smaller amplitudes than tamping screeds. In contrast to the tamping screeds, the entire bottom plate compacts the asphalt mix. A larger contact area permits faster paving speeds.
Precision asphalt laying requires modern electronic systems that automatically control the material thickness [2]. There are two such systems: the grade controller, which helps to maintain the laying thickness, i.e. the surface evenness; and the slope controller, which checks the cross-slope of the layer.

A grade controller, working off a reference surface, automatically maintains the height of the screed and the layer thickness of the material. For best results the reference surface needs to be as level as possible.

Where there is a level surface, for example a curbstone, a short control ski (approx. $30 \mathrm{~cm}$ ) can be used to sense the variations in height. Short skis are also used as a joint matcher when laying a new lane parallel to the one already in place.

Long control skis (between 3 and 9 meters) are used when the existing surface is not fully even. They ride over bumps and dips averaging out the longitudinal errors of the surface being paved. They are also known as averaging beams.

The grade controller can also work off string lines. These are rigged up when no accurate surface is available to work off, for example on new road construction projects. In open areas, such as parking 
lots, playgrounds or runways, laser systems may be used to control the screed level.

String line systems are used in conjunction with a grade controller to ensure the longitudinal evenness of the mat where there is no other appropriate surface to work off.

The slope controller maintains the specified left and right-hand cross-slope of the mat during the laying procedure. It detects any deviation of the screed from a pre-set cross-slope and generates the necessary signals to restore the original setting. The slope controller works of the screed itself, and it is attached by a mechanical link system connected to the left and right side of the screed.

An electronic slope controller mounted on a beam in front of the driver allows the screed operator to set and maintain the desired inclination percentage, for example to achieve a crown profile on wide sections or on super-elevated curves.

\section{ASPHALT MIXES - COMPACTOR INTERACTION}

The simplest asphalt mixes model is related to the compaction process when the screed in pavers or compactors is not losing contact with the material. Among the most often applied models is the KelvinVoigt model of asphalt mixes composed of a spring $K(\delta)$ and the damper $C(\delta)$, as shown in Figure 1.

When the exciter vibrates on the asphalt mix, its vibrating behavior varies with certain properties of the asphalt and with the changing physical parameters of the compaction process such as the density factor $\delta$.

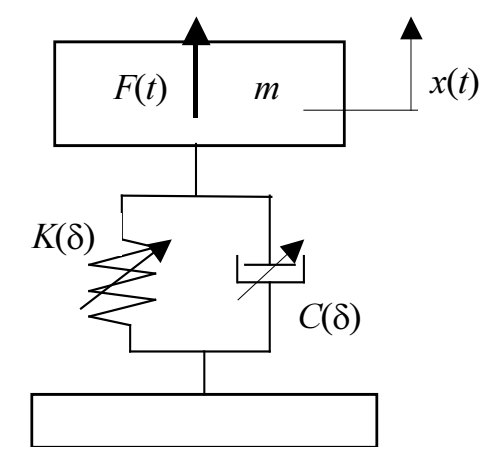

Fig. 1. Simulation model for the compactor - asphalt mixes system

The equation of motion for the compactor can be expressed as follows:

$$
\begin{gathered}
m \cdot \frac{d^{2} x(t)}{d t^{2}}+C(\delta) \cdot \frac{d x(t)}{d t}+K(\delta) \cdot x(t)=F(t) \\
F(t)=8 \cdot \pi^{2} \cdot m_{e} \cdot r \cdot f^{2} \cdot \sin (2 \cdot \pi \cdot f \cdot t)
\end{gathered}
$$

where $m$ - mass of the compactor, $x(t)$ - vertical displacement of the compactor,
$K(\delta)$ - spring constant of the asphalt mix,

$C(\delta)$ - damping coefficient of the asphalt mixes,

$m_{\mathrm{e}}$-eccentric masses in rotating mass-type excitation,

$r$ - eccentricity of eccentric masses,

$f$ - frequency of excitation,

$t$ - time.

The resonant frequency and the damping ratio of the compactor-asphalt mix system are given by the following equations:

$$
\begin{aligned}
& f_{0}=\frac{1}{2 \cdot \pi} \sqrt{\frac{K(\delta)}{m}} \\
& \zeta_{0}=\frac{C(\delta)}{2 \cdot \sqrt{m \cdot K(\delta)}}
\end{aligned}
$$

The efficiency of vibrating compaction depends upon several parameters. Among them the most important is the amplitude of compactor acceleration. From Equations (1) and (2), and taking into account Equations (3) and (4) the following equation is obtained:

$$
A_{a}=\frac{8 \cdot \pi^{2} \cdot m_{e} \cdot r \cdot f^{2}}{m} \cdot \frac{\eta^{2}}{\sqrt{\left(1-\eta^{2}\right)^{2}+4 \cdot \eta^{2} \cdot \zeta_{0}^{2}}}
$$

where the frequency ratio

$$
\eta=\frac{f}{f_{0}}
$$

Figure 2 shows a typical relationship between the acceleration amplitude (curve 1) and the sensitivity of acceleration amplitude (curve 2) versus the frequency ratio. From this figure one can observe the high level of sensitivity of the acceleration amplitude in the resonant range. This range of exciter operation is preferable from the point of view of work efficiency and energy consumption.

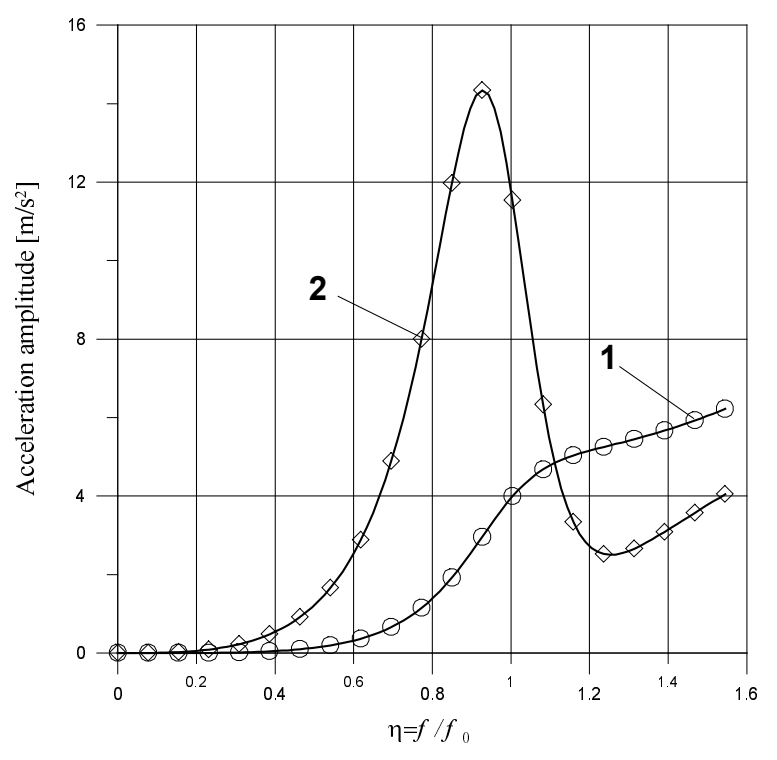

Fig. 2. Acceleration amplitude versus frequency ratio

The resonant frequency and the damping ratio are dependent upon the density factor $\delta$ and this 
relationship can be used to design an automatic control system of the asphalt compaction task.

In the case of vibrating compaction of an asphalt mix, another relationship describes the state of the compacted medium. There is a relationship between the density factor $\delta$ and the compacted material thickness $h$ for a given amplitude of force $F(t)$ by the vibration frequency $f$.

From experimental data taken into account in Equation (2) this relationship may be approximated as follows [4]:

$$
\delta=\delta_{\max }\left(1-\exp \left(-\frac{k_{\delta} \cdot f^{3}}{h \cdot v}\right)\right)
$$

where

$\delta_{\max }$ - the maximum density factor for asphalt mix,

$k_{\delta}-$ coefficient of compaction for considered compactor,

$v$-speed of compactor.

Based on Equation (6), Figure 3 shows the typical relations between the asphalt mix density factor and compaction material thickness and the change in this relationship with respect to vibration frequency.

\section{THE CONCEPT OF AUTOMATIC CONTROL}

At a given material thickness the increasing of density factor by the increasing of vibration frequency gives the possibility to create a predictive control system in the case of laser-control asphalt pavers or pavers with flexible connectors. When a thicker asphalt layer is applied, one ought to consider subsequent compaction.

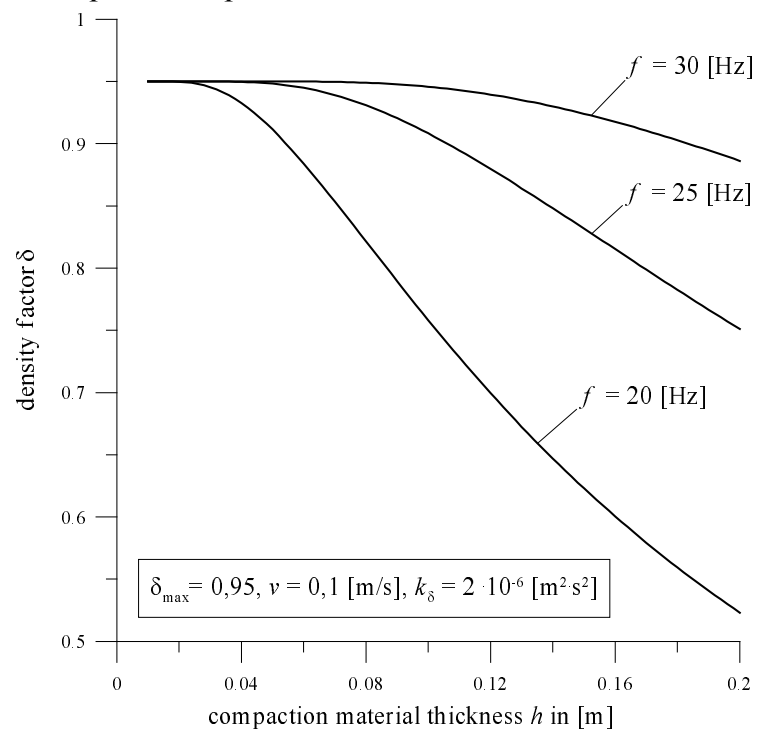

Fig. 3. Asphalt mix density factor versus compaction material thickness

To reach $\delta=$ const. if the material thickness is changed one can shift the frequency of vibration or the speed of compactor. With $v=$ const. the vibration frequency depends upon the compacted material thickness as follows [5]:

$$
f=k_{f} \cdot \sqrt[3]{h}
$$

Figure 4 shows the relationship between vibration frequency and compaction material thickness while reaching a constant density factor of the asphalt mix after compaction by $v=0.1[\mathrm{~m} / \mathrm{s}]$.

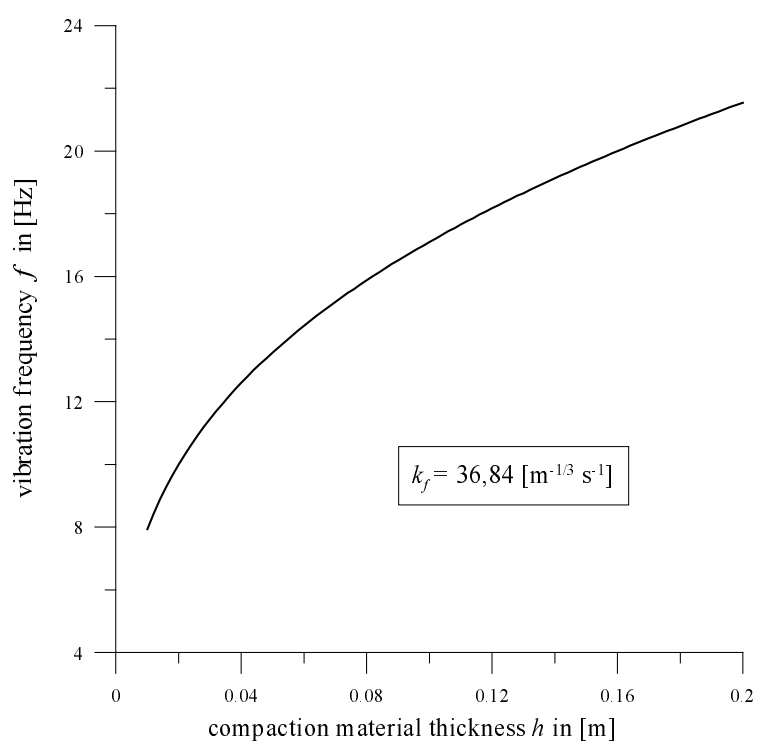

Fig. 4. Vibration frequency versus compaction material thickness

Figure 5 illustrates the general concept of correcting surface unevenness in a road construction process.

Surface 1 is the final surface of the asphalt layer, after precompaction, with density factor $\delta_{\mathrm{e}}$. The screed creates Surface 2 and the thickness of the asphalt layer is corrected. Before compaction this layer has the density factor $\delta_{\mathrm{p}}$.

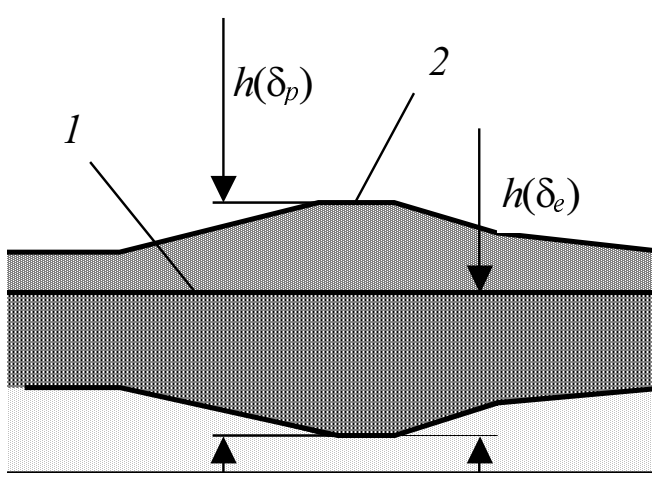

Fig. 5. Concept of correcting surface unevenness

The general structure of a predictive leveling and compacting control system is presented in Figure 6 . The automatic material thickness control system consists of two leveling transducers, $\Delta y_{\mathrm{r}}$ and $\Delta y_{\mathrm{l}}$, and of controller $R_{h}$ with two channels working 
independently of one another, with deviations from the rated value. Both channels operate electromagnetic four-way valves, which adjust the screed crossbeams over hydraulic cylinders, thus correcting the height of asphalt layers. A difference exists between the two methods of use:

1. one-sided scanning by height controller from an advanced reference line with transmission by the cross fall regulator (pendulum) to the other side. In this case we have:

$$
\begin{gathered}
\Delta h_{r}=\Delta h_{r}(s), \\
\Delta h_{l}=\Delta h_{r}+\Phi(s) \cdot L,
\end{gathered}
$$

where,

$s$ - the length of the pavers, $\Phi-$ the angle of pendulum, $L-$ the scanning base,

2. scanning on both sides by two controllers of the asphalt layer height from advanced reference lines, without the use of the cross fall regulator. In this case we have [3]:

$$
\begin{gathered}
\Delta h_{r}=\Delta h_{r}(s), \\
\Delta h_{l}=\Delta h_{l}(s) .
\end{gathered}
$$

The function of the measure, taken up by the high reference, is transmitted directly to the control valve for correcting the layer thickness according to the beginning density factor $\delta_{p}$ of the asphalt mix.

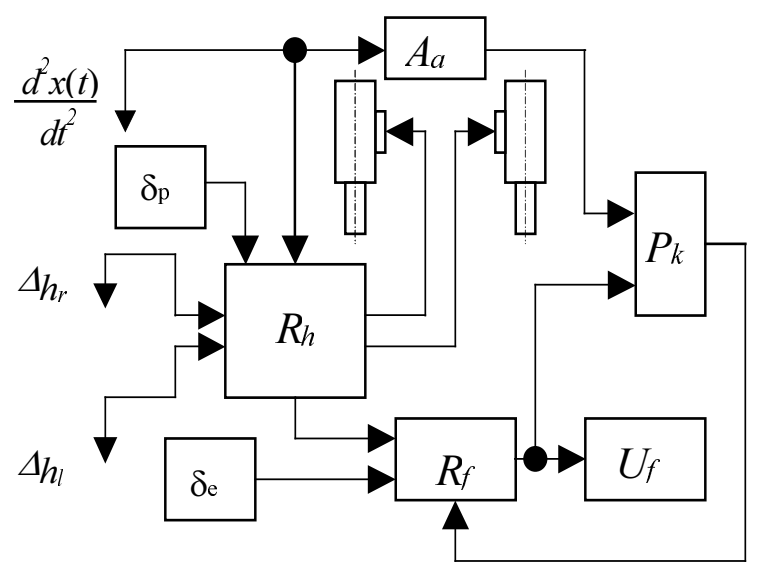

Fig. 6. The concept of the control system

The cross fall regulator (pendulum) illustrates an artificial horizon that is changeable in its position (cross-fall). This regulator controls the other side of the screed.

The controller is fixed on a transverse member between the screed crossbeams, and it is located within the driver's sight. The fastening points of the transverse member at the screed crossbeams have been selected in such a way that the best reaction from the controller is secured to insure the quality of the asphalt laying task.
With a switched-off automatic control system, the height adjustment of the screed can be achieved by the use of the manual push-button control.

The proposed compaction controller consists of the following functional units:

- the screed acceleration sensor with amplitude meter $A_{a}$,

- $\quad$ vibration frequency regulator $R_{f}$

This type of controller may monitor the compaction process with respect to the final density factor of the asphalt mix $\delta_{p}$ and produce a permanent record of all factors that influence pavement density.

Material thickness of before compaction is

$$
h\left(\delta_{p}\right)=h_{0}+\Delta h_{0}+\varepsilon+\Delta h(\varepsilon),
$$

and after compaction

$$
h\left(\delta_{e}\right)=h_{0}+\varepsilon,
$$

where

$h_{0}-$ the thickness of asphalt layer without unevenness,

$\Delta h_{0}$ - the thickness of asphalt layer reduced in compaction process,

$\varepsilon$ - the height of unevenness according to(8) and (9), $\Delta h(\varepsilon)$ - the thickness of asphalt layer reduced in compaction process when unevenness is present.

Taking into account the relationship

$$
\delta_{p} h\left(\delta_{p}\right)=\delta_{e} h\left(\delta_{e}\right)
$$

we get

$$
\Delta h_{0}=h_{0}\left(\frac{\delta_{e}}{\delta_{p}}-1\right)
$$

and

$$
\Delta h(\varepsilon)=\varepsilon\left(\frac{\delta_{e}}{\delta_{p}}-1\right) .
$$

The formula (16) describes the displacement of the paver screed to correct the laying height with respect to unevenness.

From Equations (6) and (7) we can get a new formula for frequency of vibrations:

$$
f=\left[\frac{v \cdot\left(h_{0}+\varepsilon\right) \frac{\delta_{e}}{\delta_{p}} \ln \frac{\delta_{\max }}{\delta_{\max }-\delta_{e}}}{k_{\delta}}\right]^{\frac{1}{3}} .
$$

The output signal $U_{\mathrm{f}}$ is used to control the hydraulic drive system of an exciter, equipped with variable displacement hydrostatic pump and fixed displacement motor, as shown in Figure 7.

For hydraulic drive of unbalanced vibration exciter the volumetric flow equations for the 
hydrostatic pump and the motor can be written as follows:

$$
\begin{gathered}
Q_{p}=f_{p} \cdot V_{\varphi p}-C_{V p} \cdot p-\frac{V_{0}}{B} \frac{d p}{d t}, \\
Q_{s}=f_{s} \cdot V_{\varphi s}+C_{V s} \cdot p,
\end{gathered}
$$

where:

$Q_{\mathrm{p}}-$ volumetric flow rate of the pump,

$f_{\mathrm{p}}$ - frequency of the pump rotor,

$V_{\varphi p}$ - capacity of pump,

$C_{\mathrm{Vp}}$ - coefficient of pump leakage,

$p$ - pressure,

$V_{0}$ - volume of oil between pump and motor,

$B$ - bulk modulus of the hydraulic oil,

$Q_{\text {s }}$ - volumetric flow rate of the motor,

$f_{\mathrm{s}}-$ frequency of the motor rotor,

$V_{\varphi s}$ - capacity of motor,

$C_{\mathrm{Vs}}-$ coefficient of hydraulic motor leakage.

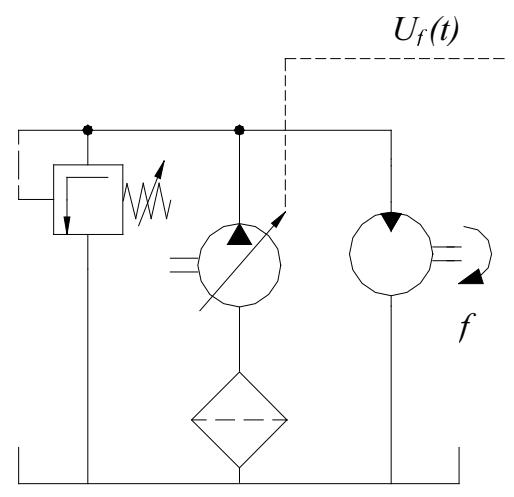

Fig. 7. The vibration frequency control system

The working pressure $p$ in the system can be calculated from formula below

$$
p=2 \pi\left(\frac{J}{V_{\varphi s}} \frac{d f_{s}}{d t}+\frac{\alpha_{s}}{V_{\varphi s}} f_{s}\right),
$$

where:

$J$ - motor and exciter moment of inertia,

$\alpha_{\mathrm{s}}-$ coefficient of motor viscous friction.

Taking into account (18), (19) and (20) and the relation $Q_{\mathrm{p}}=Q_{\mathrm{s}}$, we obtain Equation 21

$$
\frac{d^{2} f_{s}}{d t^{2}}+a \frac{d f_{s}}{d t}+b \cdot f_{s}=c \cdot V_{\varphi p}
$$

where:

$$
\begin{gathered}
a=\frac{\alpha_{s}}{J}+\frac{B\left(C_{V p}+C_{V s}\right)}{V_{0}} \\
b=\frac{B}{2 \pi J \cdot V_{0}}\left[2 \pi \alpha_{s}\left(C_{V p}+C_{V s}\right)+B \cdot V_{\varphi s}^{2}\right]
\end{gathered}
$$

$$
c=\frac{B \cdot f_{p} \cdot V_{\varphi s}}{2 \pi J \cdot V_{0}}
$$

If parameters of a vibration compaction process are changed with respect to the width of the paving layer, then the precompacting devices should be divided into sections and work individually based on the different conditions encountered.

The frequency of vibration is changed in respect to high of unevenness transform in signal $U_{\mathrm{f}}(t)$ and this signal is used to change the pump capacity

$$
V_{\varphi p}(t)=V_{\varphi p 0}+K_{P} \cdot U_{f}(t)
$$

where

$V_{\varphi p 0}$ - capacity of pump in steady - state conditions $(\varepsilon=0)$,

$K_{p}-$ the gain coefficient.

If volume elasticity of oil is negligible the Equation (21) is very simple and this fact is very important from the point of view of the structure of the vibration frequency regulator.

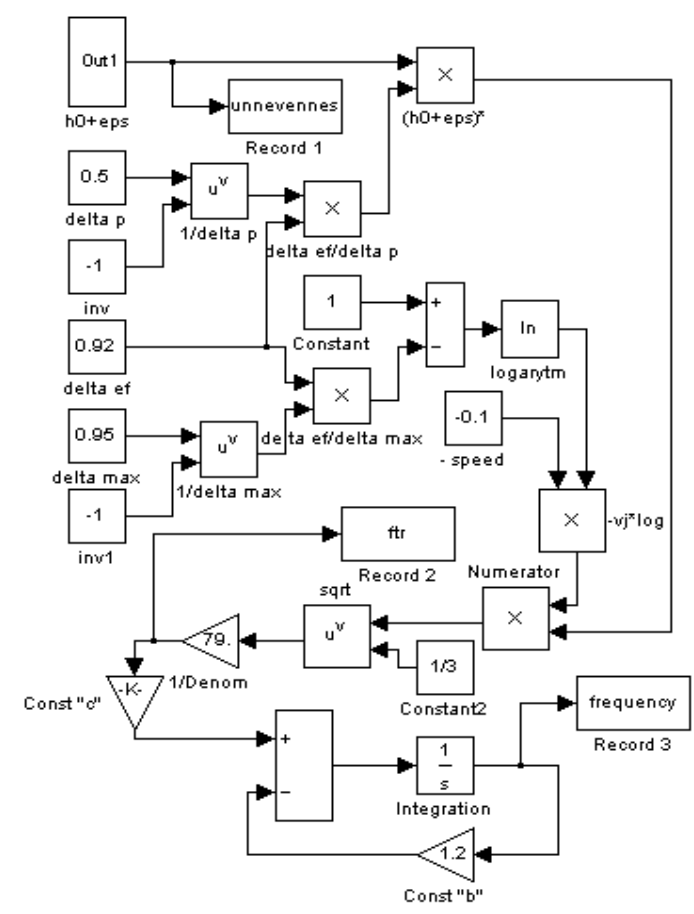

Fig. 8. Block diagram of the proposed on board controller created in MATLAB-

$$
\text { SIMULINK }^{\circledR} \text { program }
$$

Figure 8 shows the functional diagram of controller used in simulations of frequency control in the cases, when the unevenness has the form of half sinusoid and the regulator is created without considering the oil elasticity in hydraulic drive of the exciter [6].

According to the proposed procedure, the correction layer for the assumed shape of unevenness should have the form which allows for obtaining 
even surface with required compaction ratio after compaction. The longitudinal section of such an asphalt mix layer is shown at Figure 9. Presented unevenness is used as a input to the controller and so

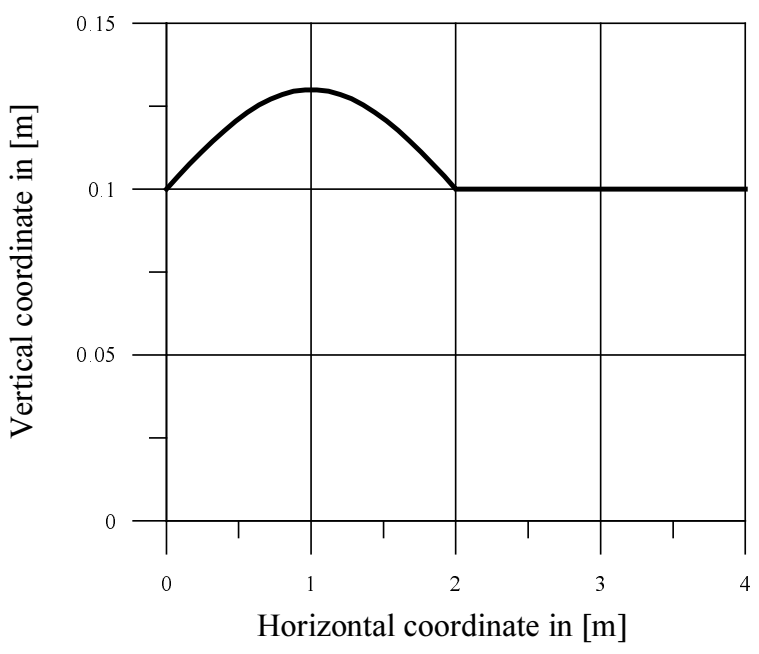

Fig. 9. The longitudinal section of correction layer before compaction

effects in adequate changes of compaction parameter - vibration frequency $f$. This is shown at Figure 10 where we can observe that the output frequency of the controller 2 follows the calculated frequency characteristic 1 with sufficient accuracy for correction compaction process.

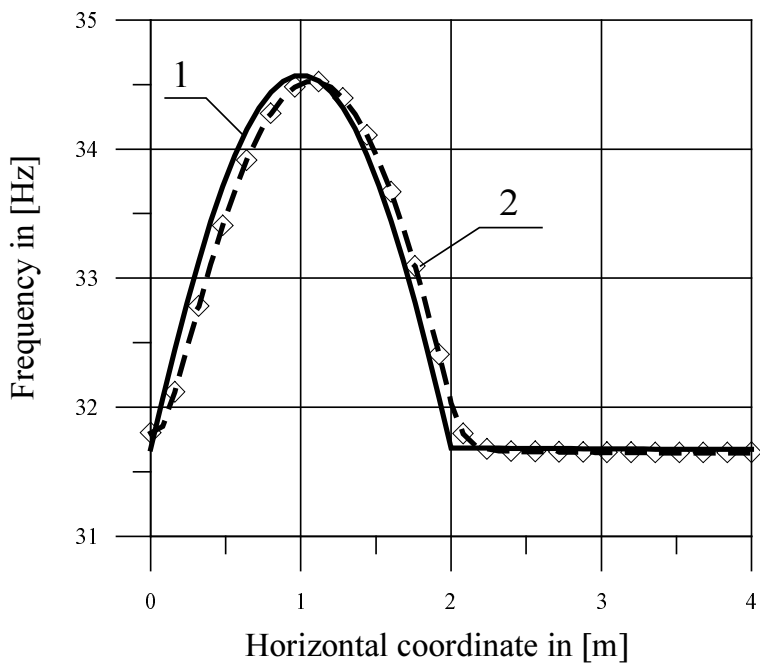

Fig. 10. The correction frequency for given unevenness compaction; 1 - calculated, 2 - from the controller

The simulation results show that the main goal of such predictive leveling and compacting control system could be reached effecting not only an even but also the same compaction ratio of the asphalt base layer which could not be corrected during next compaction processes by use typical static or/and vibration rollers thus effecting quality of the road wearing course.

\section{CONCLUSION}

Base on the discussion presented above, it is possible to correct the quality of the surface of an asphalt layer with the use of an automated control system based on the asphalt density and asphalt compaction parameters. The proposed predictive leveling and compacting control system consists of two transducers and a controller which adjust the screed crossbeams over hydraulic cylinders, correcting the laying height and the compaction controller with main functional units: the acceleration amplitude meter and frequency regulator.

It is anticipated that a full scale design and development of the proposed controller will lead to commercial applications of this concept on the existing and future asphalt pavers used in the road building industry.

\section{REFERENCES}

[1] Cheng-che Li, Thomas R.H., Oloufa A.A.: Development of an Automated Real-Time Tracing System for Pavement Compaction. $12^{\text {th }}$ ISARC Proceedings, 1995, IMBiGS, Warsaw, Poland pp. 49-54.

[2] Compaction and Paving: Theory and Practice. Dynapac Publication No. HC 1111-2 Eng., 1989.

[3] Kosiara A.: Laser Positioning of Mobile Earthmoving Machines. Manipulators. OffRoad Machines and Vehicles in Theory and Practice. Proceedings of the International Conference. Wrocław, Poland, 1996 pp. 419424.

[4] Nijboer L.W.: Plasticity as a factor in the design of dense bituminous road carpets. Tokyo, Japan, 1967.

[5] Tateyama K., Nakajima S., Fujiyama T.: The evaluation of ground properties and its application to the automatic control of vibratory soil compactors. Proceedings, $12^{\text {th }}$ ISARC, IMBiGS, Warsaw, Poland, 1995, pp. 563 - 570,

[6] Dorf R.C., Bishop R.W.; Modern Control Systems. Addison-Wesley Publishing Company, Inc. Seventh Edition, 1995. 